$\begin{array}{cccccr}\text { Argument } & \text { For ci(x) } & \text { Read ci(x) } & \text { Argument } & \text { For ci(x) } & \text { Read ci(x) } \\ 0.057 & -2.288200 & -2.288300 & 2.34 & 0.335436 & 0.335434 \\ 0.512 & -0.157037 & -0.157039 & 2.38 & 0.326406 & 0.323405 \\ 0.656 & +0.049348 & +0.049948 & 2.39 & 0.320358 & 0.320356 \\ 0.843 & 0.233949 & 0.233943 & 2.45 & 0.301748 & 0.301746 \\ 1.67 & 0.468998 & 0.468996 & 3.91 & -0.125351 & -0.125349 \\ 1.68 & 0.463377 & 0.468375 & & & \\ 1.69 & 0.467701 & 0.467699 & 8.55 & +0.095875 & +0.095785\end{array}$

The argument following 2.58 should read 2.59, not 2.69; the argument following 5.74 should read 5.75 , not 3.75 .

Apart from the 13 errors greater than one unit in the last place, listed above, there were 334 last-place unit errors in values of $c i(x)$, and 127 such errors in si(x).

312 Schenectadiy Ave.

ARNold N. Lowan

Brooklyn 13, N. Y.

167.-J. Travers, "Perfect numbers," Math. Gazette, v. 23, 1939, p. 302.

The 10-th and 11-th perfect numbers are given incorrectly. The 30-th digit (from the left) of $P_{10}$ should be 4, not 2. The 27-th digit of $P_{11}$ is missing; it should be 4 .

206 Spring Street

HORACE S. UhLER

Meriden, Connecticut

\title{
UNPUBLISHED MATHEMATICAL TABLES
}

EDITORIAL Note: Beginning with this issue we are starting a collection of unpublished mathematical tables to be known as the UMT FILE. Authors of tables which have no immediate prospect of publication are invited to submit copies for deposit in UMT FILE. Description of such tables will appear in UMT and photostat or microphilm copies will be supplied at cost to any reader of $M T A C$. Address tables or correspondence to D. H. LEHMER, 942 Hilldale Ave., Berkeley 8, California.

84[C].-G. W. Reitwiesner, Arccot 5 and arccot 239 to 2035 places. On deposit in UMT FILE.

This is a by-product of the author's calculation of $\pi[M T A C, \mathbf{v} .4, \mathrm{p} . \mathbf{x x}]$.

85[F].-D. Jardan [Yarden] \& A. Katz. Additional page 477 to D. N. Lehmer's Factor Table. On deposit in UMT FILE.

This single page is of the same form as Lehmer's well known factor table [Carnegie Institution of Washington, Publ. 105, 476 p.] and covers the range $10017000-10038000$.

86[F].-Sylvester Whitten. Tables of the totient and reduced totient function. Manuscript deposited in UMT FILE.

The totient function, $\phi(n)$, is the number of numbers not exceeding $n$ and relatively prime to $n$. The function $\phi(n)$ has the property that $a^{\phi(n)}-1$ is divisible by $n$ for all $a$ prime to $n$. The reduced totient $\psi(n)$ is defined as the least positive number $m$ such that $a^{m}-1$ is divisible by $n$ for all $a$ prime to $n$ so that $\psi(n) \leqslant \phi(n)$. 
Table 1 gives $\phi(n), \psi(n)$, and $\Phi(n)=\phi(1)+\phi(2)+\cdots+\phi(n)$ for $n=1(1) 1200$, and the approximation $3 n^{2} \pi^{-2}$ to $\Phi(n)$ for $n=1000$ (1)1200 to 2D. The author notes that $\Phi(1063)=344116>3(1063)^{2} \pi^{-2}=344115.92$, contrary to a conjecture of J. J. SYLVESTER.

Table 2 gives $s(n)=\frac{1}{2} n \phi(n)$, the sum of the numbers $\leqslant n$ and prime to $n$. Also $S(n)=s(1)+s(2)+\cdots+s(n)$, and the approximation $n^{3} \pi^{-2}$ to $S(n)$ to $2 \mathrm{D}$ for $n=1(1) 100$.

Editorial Note. The late Mr. Whitten, a telephone engineer, became interested in the above functions in connection with the problem of splicing telephone cables to minimize cross-talk. [See H. P. LAwTHER, "An application of number theory to the splicing of telephone cables," Am. Math. Monthly, v. 42, 1935, p. 81-91]. The above tables extend those of J. J. Sylvester and A. CAUCHY, see Guide to Tables in the Theory of Numbers, National Research Council, Bulletin, no. 105, 1941, p. 6, 7. [MTAC, v. 3, p. 531].

87[G, I].-H. E. SAlzer, Derivatives of $[y(x)]^{n}$, Manuscript in the possession of the author.

This manuscript gives the explicit expressions as polynomials in $y$ and its derivatives for the first twelve derivatives of $[y(x)]^{n}, n=2(1) 20$. The expression $d^{m}[y(x)]^{n} / d x^{m}$ is a sum of $m$ terms, each term consisting of two factors, one factor depending only on $n$, the other factor depending only on $m$. The manuscript is in the form of two separate superimposable tables, which enables the user to place in juxtaposition the two factors corresponding to each of the $m$ terms of $d^{m}[y(x)]^{n} / d x^{m}$.

1903 Ocean Ave.

H. E. SAlzer

Brooklyn, 30, N. Y.

88[L].-R. T. BIRGE, Table of Fresnel Integrals, 3 mimeographed sheets, Dept. of Physics, University of California, Berkeley. Copy in UMT FILE.

The table gives the Fresnel integrals

$$
C(u)=\int_{0}^{*} \cos \left(\frac{1}{2} \pi x^{2}\right) d x \text { and } S(u)=\int_{0}^{*} \sin \left(\frac{1}{2} \pi x^{2}\right) d x
$$

for $u=[0(.05) 12.05 ; 4 \mathrm{D}]$. These tables extend those of C. M. Sparrow beyond $u=8$. For reference to these and other tables of Fresnel integrals see $M T A C$, v. 3, p. 479 , v. 4 , p. 58 .

89[L].-CARL Hammer, Systems of particular solutions of the differential equation $y^{(n+1)}+y=0$, and numerical tables, manuscript in the possession of the author, Walter Harvey Junior College, N. Y., and of the Library at Brown University, 6 sheets.

The functions tabulated are

$$
H_{n k}(x)=\sum_{r=0}^{\infty} \frac{(-1)^{r} x^{(n+1) r+k}}{[(n+1) r+k] !}(k=0,1,2, \cdots, n) .
$$

These solutions are given explicitly in terms of trigonometric and hyperbolic functions for $n=0(1) 5$. Actual values of the functions are given to $5 \mathrm{D}$ for $n=2(1) 5$ and for $x=-5(.1) 5$. 